\title{
How to master the host immune system? Leishmania parasites have the solutions!
}

\author{
Matteo Rossi and Nicolas Fasel \\ Department of Biochemistry, University of Lausanne, Chemin des Boveresses 155, Epalinges CH-1066, Lausanne, \\ Switzerland \\ Correspondence to: N. Fasel; E-mail: nicolas.fasel@unil.ch
}

Received 30 November 2017, editorial decision 19 December 2017; accepted 21 December 2017

\begin{abstract}
Infection by protozoan parasites of the genus Leishmania results in the development of leishmaniasis, an increasingly prevalent group of diseases affecting over 12 million people worldwide. Leishmaniasis can have very different outcomes ranging from cutaneous lesions, mucosal lesions to visceralization depending on the species of the infecting parasite and on the immune response developed by the host. As an obligate intracellular parasite, residing within macrophages, Leishmania evolved in strict contact with the host immune system, developing different mechanisms to evade or modulate the immune response. Various types of immune responses are observed during different Leishmania spp. infections, resulting in parasite clearance but also contributing to the pathogenesis, thus increasing the complexity of the course of the disease. Interestingly, depending on the type of leishmaniasis developed, opposite treatment strategies, which either boost or inhibit the inflammatory response, have shown efficacy. In this review, we summarize the contribution of different immune cell types to the development of the antileishmanial immune response and the parasite strategies to evade and modulate host immunity. Further, we discuss the involvement of co-infecting pathogens in the determination of the outcome of leishmaniasis and on the effectiveness of treatment and the implication of the immune response for treatment and vaccine development.
\end{abstract}

Keywords: co-infection, immune modulation, leishmaniasis

\section{Introduction}

Infection with the protozoan parasite Leishmania can lead to the development of leishmaniasis, a prominent but neglected disease affecting over 12 million people worldwide, with an increasing geographical extension, limited treatment options and still no human vaccine $(1,2)$. Depending principally on the species of the infecting parasite, leishmaniasis can have a wide spectrum of manifestations (2). Cutaneous leishmaniasis (CL) is most frequently displayed as ulcerating skin lesions at the site of the sand fly bite, and caused by species such as L. major, L. braziliensis or L. guyanensis $(2,3)$.

CL can evolve into mucocutaneous cutaneous leishmaniasis (MCL) or disseminated cutaneous leishmaniasis (DCL), two more severe forms of the disease characterized by parasite migration to secondary sites and the formation of metastatic lesions $(4,5)$. Interestingly, these secondary lesions often present very low parasite numbers, but concomitant exaggerated inflammation provokes tissue destruction. This dissemination and re-activation of the infection at secondary sites can occur years after the primary infection and are observed with species such as L. braziliensis, L. guyanensis and $L$. aethiopica. Despite being less frequent than $C L$, visceral leishmaniasis $(\mathrm{VL})$, caused by $L$. donovani and $L$. infantum, is the most dangerous manifestation and is characterized by parasite dissemination to the liver and spleen that can be fatal if left untreated.

Aside from the species of the infecting parasite, the outcome of leishmaniasis is mainly determined by the host immune response and several immune-related genetic polymorphisms have been associated with the severity of the disease. Leishmania being obligate intracellular parasites, after the transmission to humans by a sand fly bite, they need to rapidly locate their host cells. In the mammalian host, Leishmania reside mainly in long-lived resident macrophages where they differentiate from promastigotes to amastigotes and then survive in the hostile, acidic and higher temperature environment of a phago-lysosome-like organelle. To establish the infection, some (but not all) Leishmania species require the recruitment of inflammatory monocytes, macrophages 
and neutrophils at the infection site. Once inside its host cell, Leishmania hide from the immune system to survive. Thus, Leishmania had to develop different strategies to shape the immune response from its very beginning, affecting both innate and adaptive immunity to promote its own survival.

Considering the abundance of excellent recent reviews on leishmaniasis, we will focus our review on some of the actual knowledge of innate and adaptive immune responses to Leishmania and parasite evasion techniques, then address the effect of co-infections on leishmaniasis and finally analyze the implications for treatment and vaccine development.

\section{Innate immunity to Leishmania}

The first hours after the infection are crucial for obligate intracellular Leishmania parasites to rapidly localize phagocytes to colonize its host. At this initial stage, Leishmania benefit from pro-inflammatory properties of the sand fly saliva that plays an essential role in phagocyte chemo-attraction, being thus an important determinant of infection outcome (6). The early phases of Leishmania infection are also affected by the dual role of complement, as complement-mediated lysis eliminates parasites whereas opsonization promotes parasite up-take by phagocytes, thus favoring the infection. The Leishmania lipophosphoglycan (LPG) coat protects the parasite from complement-mediated lysis and at the same time induces complement activation, thus promoting parasite phagocytosis (7). Moreover, leishmanial LPG delays the formation of phago-lysosomes and allows Leishmania parasites to differentiate into its intracellular form, the amastigote (8).

\section{Leishmania and macrophages}

The long-lasting Leishmania infection is established within macrophages (phagocytic cells usually specialized in the elimination of intracellular pathogens) confining the parasite in a phago-lysosome organelle filled with lytic enzymes and low $\mathrm{pH}$. In the first hours of the infection, the most effective anti-Leishmania response of the macrophages is the production of reactive oxygen species (ROS) and reactive nitrogen species (RNS) $(9,10)$, a tightly regulated process that aims to kill any invading pathogen without damaging the host cell. This process is in part activated by phagocytosis and involves several enzymes. The two major producers of reactive species in macrophages are NADPH oxidase 2 (NOX2) and inducible nitric oxide synthase (iNOS), which produce superoxide $\left(\mathrm{O}_{2}^{-}\right)$and nitric oxide (NO), respectively $(11,12)$.

Evidently, Leishmania parasites have developed various strategies to interfere with this process (13) (see Fig. 1), such as the LPG shield that protects parasites from ROS and RNS and subsequently delays the assembly of NOX2 at the surface of the phago-lysosome in the phagocytic cup, thus suspending the production of $\mathrm{O}_{2}^{-}$(14). This process is further exploited by amastigotes, because of a thick layer of LPG (15). Further, Leishmania parasites can secrete or induce macrophages to produce arginase, which competes with iNOS for arginine and produces essential nutrients for the parasites such as L-ornithine for the synthesis of polyamines and urea, and at the same time diminishes the production of parasitotoxic NO (16). Oxidative stress could also be further

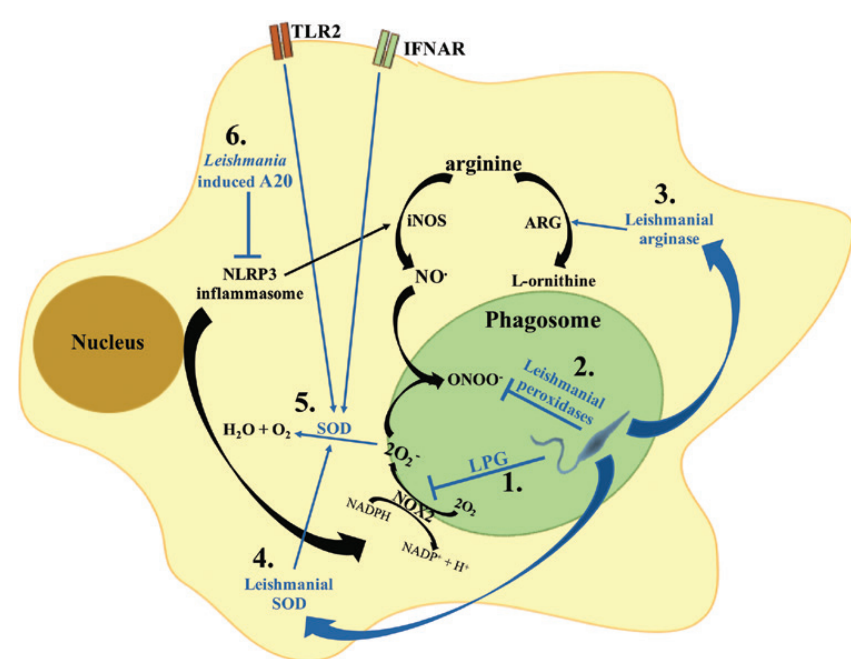

Fig. 1. Mechanisms of Leishmania interference with the macrophage oxidative stress response. Leishmania parasites have developed different strategies to interfere with the macrophage oxidative stress response. (1) Early after phagocytosis, the leishmanial LPG coat inhibits the assembly of the NOX2 complex at the phagosome membrane, thus blocking superoxide production $\left(\mathrm{O}_{2}^{-}\right)$. (2) Leishmania secrete a series of peroxidases which detoxify parasitotoxic peroxynitrite ( $\left.\mathrm{ONOO}^{-}\right)$. In addition, Leishmania parasites can secrete arginase (3), which promotes the conversion of arginine into urea and ornithine used for parasite metabolism, and secrete SOD homologues (4), which increase the detoxification of parasitotoxic superoxide. (5) Additionally, stimulation of TLR2 or the type I interferon receptor (IFNAR) during Leishmania infection induces macrophages to up-regulate SOD expression. (6) NLRP3 inflammasome activation was shown to promote the production of ROS and RNS; Leishmania inhibits inflammasome activation via the induction of the deubiquitinating protein A20, which inhibits IL-1 $\beta$ maturation.

inhibited by the leishmanial metalloprotease gp63, which interferes with macrophage signaling pathways leading to NOX2 and iNOS induction (17).

Interestingly, L. braziliensis and L. amazonensis have been shown to increase macrophage superoxide dismutase (SOD) expression, through the induction of type I interferons, thus favoring parasite survival (18), possibly acting in concert with various leishmanial antioxidant analogues present in the parasite (19-21).

In conclusion, Leishmania parasites actively master the oxidative stress from their initial entry to their survival in the macrophage.

\section{Leishmania and neutrophils}

Beside macrophages, Leishmania parasites can also be trapped by neutrophils that are rapidly recruited to the infection site, where they produce 'neutrophil extracellular traps' (NETs). These are a network of extracellular fibrils composed of DNA associated with antimicrobial proteins such as elastase, histones and toxic granules.

Despite usually being very short-lived immune cells, the neutrophil life-span highly expands during inflammation. However, the impact of neutrophils on leishmaniasis outcome is likely species-specific (22). In fact, while L. amazonensis parasites are efficiently killed by NETs, L. donovani and $L$. infantum are resistant and may even benefit from NETs formation (23). Moreover, the rapid recruitment of neutrophils 
during L. mexicana infection contributes to disease progression and chronicity (24). Further, an increased recruitment of neutrophils was observed in mice co-infected with L. major and lymphocytic choriomeningitis virus (LCMV), which developed more severe lesions compared to controls infected with just L. major (25). The molecular signatures or mechanisms underlying these differences in the relation between neutrophils and different Leishmania species are not yet known and would deserve thorough investigations.

\section{Leishmania and inflammatory monocytes}

Not only resident macrophages and neutrophils, but also inflammatory monocytes, recruited via platelet activation very early at the infection site, can be infected by Leishmania parasites, as was shown for L. major (26). In general, recruitment of inflammatory monocytes correlates with increased resistance to $L$. major infection (26). Inflammatory monocyte recruitment depends on chemokine (C-C motif) ligand 2 (CCL2/ MCP-1), which is produced by cells at the infection site upon activation via platelet-derived growth factor (26). The importance of CCL2 and recruitment of inflammatory monocytes at the site of infection was investigated in $\mathrm{C}-\mathrm{C}$ chemokine re-

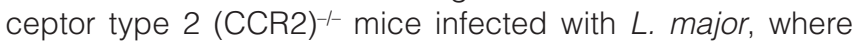
a non-healing phenotype was observed in a mouse strain usually controlling Leishmania infection. Thus, recruitment of inflammatory monocytes correlates with increased resistance to L. major infection (26).

Interestingly, monocytes are crucial to control the re-infection of resistant mice with L. major, as they clear the parasites through the production of ROS and RNS following activation by resident memory $\mathrm{CD}^{+}{ }^{+} \mathrm{T}$ cells $(27,28)$. However, this phenotype is parasite species specific, as shown following infection with $L$. amazonensis, where the recruitment of inflammatory monocytes was associated with increased parasite burden and pathology (29). Similarly, infection of human monocytes by different Leishmania species alters their adherence to connective tissues (30) and could favor dissemination of the infection.

\section{The paradoxical role of pattern recognition receptors}

A common characteristic of the different innate immune cells is the expression of pattern recognition receptors (PRRs), capable of recognizing pathogen-associated molecular patterns (PAMPs). These latter are broadly conserved motifs covering bacterial endotoxins, lipopeptides and peptidoglycans, together with single-stranded (ss) and double-stranded (ds) nucleic acids. These PAMPs are recognized via Toll-like receptors (TLRs), cytosolic DNA sensors (CDSs), NOD-like receptors (NLRs), RIG-l-like receptors (RLRs) and C-type lectin receptors (CLRs). Because of the numerous ligands expressed by the parasite $(31,32)$, the most studied PRRs are TLRs, which play different and often species-specific roles during Leishmania infection, adding complexity and diversity to their role in leishmaniasis but possibly explaining different clinical outcomes ranging from clearance to exacerbated pathology (see Fig. 2).

For example, TLR2 promotes the clearance of L. major, L. aethiopica or L. mexicana infection by promoting $\mathrm{NO}$ and ROS production, therefore favoring the development of a protective immune response in mice (33-35). However, TLR2 stimulation has also been shown to promote the persistence of $L$. braziliensis and $L$. amazonensis infection by inducing the expression of SOD1 through a PKR-IFN- $\beta$ axis (36). These contradictory roles allegedly depend either on the thickness of the LPG coat, which vary considerably between parasite species or on other molecules still to be identified.

One report suggests that TLR3 is required for the phagocytosis of $L$. donovani and the subsequent production of parasitotoxic $\mathrm{NO}$ and $\mathrm{TNF}-\alpha$ by macrophages, thus

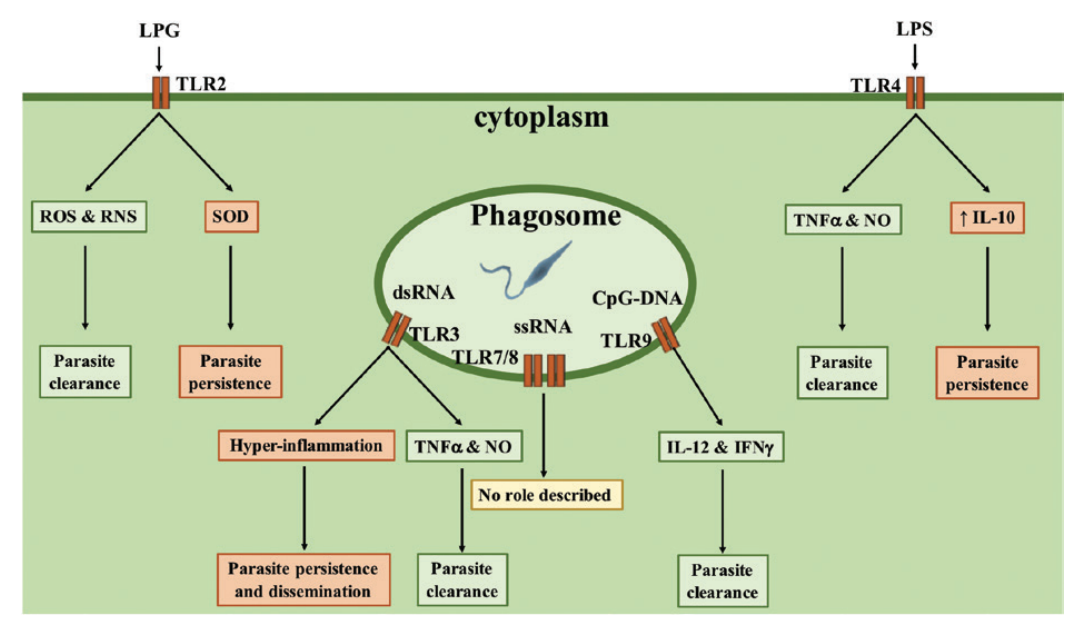

Fig. 2. TLRs' influence on the outcome of Leishmania infection. Several TLRs are stimulated during Leishmania infection and can have opposite consequences on the outcome of the infection depending on parasite species and timing of stimulation. During L. major infection, TLR2 activation increases the production of ROS and RNS, thus favoring parasite clearance, whereas in $L$. braziliensis infection it promotes the expression of SOD, leading to parasite persistence. TLR4 stimulation post-infection promotes TNF- $\alpha$ and NO production, increasing parasite killing, whereas stimulation prior to infection increases IL-10 production, favoring parasite persistence. TLR3 stimulation increases host resistance to L. donovani infection, by promoting TNF- $\alpha$ and NO production, whereas it promotes L. guyanensis virulence, by provoking a hyper-inflammatory response and the dissemination of parasites. TLR9 is the only TLR member which was solely associated with host protection, as it promotes IL-12 and IFN- $\gamma$ production by dendritic cells, thus favoring the development of a protective $\mathrm{T}_{\mathrm{h}} 1$ immune response. 
promoting parasite clearance despite the source of the ligand being unknown (37). In contrast, TLR3 stimulation was shown to induce a hyper-inflammatory response associated with increased symptoms and parasite burden during infection with L. guyanensis (38). This exacerbated phenotype is associated with the presence of an endosymbiotic virus offering its dsRNA genome to induce TLR3-dependent hyper-inflammation, suggesting that TLR3 activation by viral dsRNA genomes present in co-infection could exacerbate Leishmania-dependent pathology.

TLR4 also shows a dual role, depending on the time of stimulation. Protective TNF- $\alpha$ and NO production is observed when TLR4 is stimulated at the same time or after Leishmania infection $(39,40)$, whereas stimulation prior to infection was associated with increased IL-10 production and parasite persistence (41).

Thus far, only TLR9 has been systematically associated with resistance to various Leishmania species infections, possibly by stimulating IL-12 production by plasmacytoid dendritic cells, which in turn induces IFN- $\gamma$ production by NK cells (42-46). IFN- $\gamma$ then acts on infected macrophages and induces the production of parasitotoxic nitrites.

In conclusion, taken together, information gathered recently on the role of TLRs suggests that they can play opposite roles in Leishmania infections, depending on the infecting species and possibly on additional ligands of bacterial or viral origins acting before, during or after the infection.

While currently there is no evidence of an involvement of other TLRs, RLRs and CDSs in Leishmania infection, the studies on NLRs and CLRs are still rare and present controversial results. In fact, NLRP3 activation and IL-1 $\beta$ production were shown to protect the host against $L$. donovani, $L$. amazonensis and $L$. braziliensis, via induction of parasitotoxic oxidative stress (47). Interestingly, L. donovani promotes macrophage expression of A20 (48) which inhibits NLRP3 inflammasome activation (49) and the production of IL-1 $\beta$ (see Fig. 1). On the other hand, NLRP3 activation is detrimental in mouse infection with a unique $L$. major strain isolated from a chronic CL patient, as it promoted the development of chronic infection by increasing neutrophil recruitment at the site of infection and inducing the inflammasome and the production of IL-1 $\beta$ (50). One should be, however, careful in the interpretation of several studies implying a role for the inflammasome since, in several instances, inflammasome activation was obtained by lipopolysaccharides (LPS) stimulation concomitant with Leishmania infection.

Regarding CLRs, Dectin-1 and mannose receptor stimulation were shown to promote macrophage microbicidal oxidative stress production, favoring the clearance of $L$. infantum infection (51). In contrast, SIGNR3 was shown to promote $L$. infantum infection, by inhibiting IL-1 $\beta$, whereas Mincle was shown to promote the infection of $L$. major, by dampening dendritic cell priming $(51,52)$. Defining the molecular pathways downstream of the CLRs will be challenging but could help to further define their relevance.

\section{Adaptive immune responses to Leishmania}

The early phase after Leishmania infection is crucial to determine the outcome of the disease. In fact, once the parasite is phagocytized, innate immune cells react by producing cytokines that shape the development of adaptive immunity, thus generating either a protective or detrimental type of response. Interestingly, despite being indispensable for the complete clearance of the infection and to establish a long-lasting memory response conferring protection against re-infection, adaptive immune cells have also been shown to participate in the pathogenesis of leishmaniasis, causing tissue destruction and inducing disease relapse.

\section{CD4 T cells}

Interestingly, the murine model of $L$. major infection was originally used to define $T_{h} 1 / T_{h} 2$ T-cell polarization. In fact, $T_{h} 1$ response, characterized by the production of IFN- $\gamma$ and TNF- $\alpha$ which induce the activation of the macrophage oxidative stress machinery, induces host resistance, whereas $T_{h} 2$ response, characterized by IL-4 and IL-13 production, leads to a susceptible phenotype, unable to control Leishmania infection. However, in the last years, it became clear that this correlation is less strict in humans, and a more complex scenario has emerged also in the mouse model. In fact, DCL patients often show high levels of TNF- $\alpha$ in their lesions, suggesting that a too robust $T_{h} 1$ response and the consequent hyper-inflammatory state can favor the development of metastatic infection (53).

Moreover, other subsets of $T_{h}$ cells have been implicated in leishmaniasis outcome, such as the IL-17-producing $T_{h} 17$ $T$ cells, which have a controversial, species-specific role. Often involved in autoimmune disorders, $\mathrm{T}_{\mathrm{h}} 17 \mathrm{~T}$ cells have been associated with healing in $L$. infantum and $L$. braziliensis infection $(54,55)$, whereas they worsen the outcome of L. major- and L. guyanensis-induced leishmaniasis (56, 57). Interestingly, in this latter case, IL-17 is associated with parasite dissemination and the metastatic infectious lesion formation in the absence of IFN- $\gamma$, both in humans and mice and is correlated with the presence of a viral endosymbiont. Finally, IL-10-producing $T_{\text {reg }}$ cells have been associated with parasite latency, treatment resistance and disease relapse in Leishmania infection, both in mice and in humans (58-61).

\section{CD8 T cells}

The role of CD8 T cells during Leishmania infection seems to depend on the form of leishmaniasis, and ranges from protective to disease exacerbating (62). In the murine model of $C L$ induced by L. major, CD8 T cells are essential to control low-dose infection, whereas they are non-essential after highdose inoculation (63). In humans, higher numbers of CTLs have been observed during the acute and healing phase of L. major infection (64-66), allegedly promoting parasite clearance through the higher production of IFN- $\gamma$. CD8 T cells have a protective role also against $V L$ caused by $L$. donovani and $L$. infantum, both in the murine model and in humans, as CTLs were shown to be essential to the proper formation of granulomas. Further, CD8 T cells were associated with resistance to $L$. infantum re-infection, implying a potential use for vaccine development.

Contrarily, higher numbers of IL-10-producing CD8 T cells have been observed in DCL and post-kala-azar dermal leishmaniasis (PKDL) patients (67-69), suggesting their involvement in the dissemination of the disease. Similarly, high numbers of granzyme-positive and IL-10-refractory CTLs 
were associated with tissue destruction and disease progression in MCL patients $(70,71)$. Moreover, in the murine model, CTLs have been recently associated with increased severity and infectious metastasis development during $L$. braziliensis infection (72), and NKG2D+ CD8 T cells have been shown to increase the severity of $L$. major infection following LCMV coinfection $(25,73)$.

\section{B cells}

$\mathrm{B}$ cells are thought to play a negligible role in the pathology of leishmaniasis as Leishmania are obligate intracellular parasites. However, a few studies have reported a disease exacerbatory role of $B$ cells during $L$. donovani and $L$. amazonensis infection $(74,75)$. In fact, IgM transmembrane domain deficient ( $\mu \mathrm{MT})$ mice-which lack mature $B$ cells_clear L. donovani infection faster than wild-type (WT) mice do, because of enhanced granuloma formation and an increased $T_{h} 1$ immune response $(74,76)$. Nevertheless, $\mu \mathrm{MT}$ mice show more severe liver necrosis compared with WT mice, because of increased recruitment of neutrophils. Similarly, B-cell-deficient mice develop delayed and less severe lesions following $L$. amazonensis infection (75). In contrast, thus far, B cells play no role during $L$. guyanensis infection (77).

\section{Leishmania and co-infection}

Differently from the highly sterilized animal models used in research, the human body is constantly in contact with a multitude of microorganisms, which shape the immune response. In fact, commensal bacteria modulate L. major infection, as germ-free mice developed a higher parasite burden but reduced lesions, through an $\mathrm{IL}-17$-dependent mechanism (78), whereas the disruption of the microbiota skin flora exacerbates inflammation and lesion development during $L$. major infection (79).

The most intriguing pathogen-Leishmania co-infection is the presence of Leishmania RNA virus (LRV) within certain species of Leishmania parasites, which was recently described to modulate the severity of $\mathrm{CL}$ induced by $L$. guyanensis and $L$. braziliensis $(5,38)$. In fact, LRV has been associated with the development of DCL, as viral dsRNA recognition by TLR3 improves infected macrophage survival by phosphorylation of the pro-survival kinase Akt (77) and is the driving force in the dissemination of the infection (57). The presence of a second species of LRV within L. major and more recently in $L$. aethiopica, another species known to cause $\mathrm{DCL}$, suggests an even greater role of LRV in the determination of Leishmania infection (80). Moreover, the pathologyincreasing effect of LRV completely relies on production of type I interferons, implying that other viral co-infections could have the same effect (81).

Of great interest are also the reports of sand fly bearing both Leishmania parasites and different Phleboviruses, which could thus be transmitted together in a simultaneous coinfection (82-84). Interestingly, co-infection with the Toscana phlebovirus increases both parasite burden and lesion size in a murine model of $L$. guyanensis infection (81). Interestingly, the presence of Narna-like virus 1-a virus infecting Leptomonas seymouri (protozoan parasites also transmitted by sand flies) - has recently been reported in $L$. donovani isolates from India, underscoring the existence of triple coinfection and suggesting its involvement in the development of PKDL (85).

Currently, HIV is still the most studied co-infecting pathogen as it worsens both $V L$ and $C L$, inducing the development of atypical symptoms and increasing treatment resistance and disease relapses (86-88). In fact, HIV infection decreases CD4 T-cell numbers, favors a $\mathrm{T}_{\mathrm{h}} 2$ immune response and impairs macrophages' functions, thus promoting parasite persistence and disease development (89-91). Interestingly, mice co-infected with LCMV, both before or after L. major infection, develop bigger leishmanial lesions, through a mechanism involving the increased inflammation generated by bystander CD8 T-cell activation $(25,73)$. Moreover, LCMV co-infection increases lesion size and parasite burden, and promotes parasite dissemination and disease relapse of L. guyanensis-induced leishmaniasis (81). In this second case, the mechanism completely relies on production of type I interferons and is associated with the inhibition of macrophage responsiveness to IFN- $\gamma$.

\section{Implications for treatment and vaccine development}

The current leishmaniasis treatment strategy focuses solely on parasitotoxic drugs, neglecting the importance of the host immune response. As leishmaniasis is an immune-mediated disease, immunotherapy could be crucial for the development of new treatments or to improve the efficacy of the current ones. In particular, because of the central role of TLRs in the initiation of the anti-leishmanial immune response, the use of TLR agonists or antagonists could help to improve the efficacy of current drugs. For instance, combination treatment with the synthetic bacterial lipopeptide Pam3Cys (a TLR2 ligand) was shown to improve the efficacy of miltefosine in a murine model of $\mathrm{VL}$, by promoting a $T_{h} 1 \mathrm{immune}$ response and ROS and RNS production (92). Further, TLR9 stimulation with D-type CpG reduced lesion severity and parasite burden in Leishmania-infected rhesus macaques (93).

Interestingly, D-type CpG treatment protected also simian immunodeficiency virus (SIV)-infected rhesus macaques from Leishmania infection, suggesting its possible application in Leishmania-HIV co-infected human patients. Moreover, the use of a topical formulation of Imiquimod (a TLR7 ligand that induces a potent pro-inflammatory response) in combination with antimony was shown to reduce the healing time and improve wound repair in Peruvian CL patients (94), setting the stage for the simultaneous use of TLRs agonists and anti-Leishmania drugs as CL therapeutics. Paradoxically, a completely opposite strategy-the use of pentoxifylline (a TNF- $\alpha$ inhibitor used to treat autoimmune disorders) in combination with antimony-improved healing and reduced the relapse rate in MCL patients (95). However, the use of TNF- $\alpha$ blockers was also associated with disease re-activation in VL patients, and thus should be carefully monitored as a therapeutic approach (96).

The development of a leishmanial vaccine remains a major challenge. Despite the efforts and different strategies tested, ranging from leishmanization to recombinant or DNA vaccines, there is still no effective vaccine, with the possible 

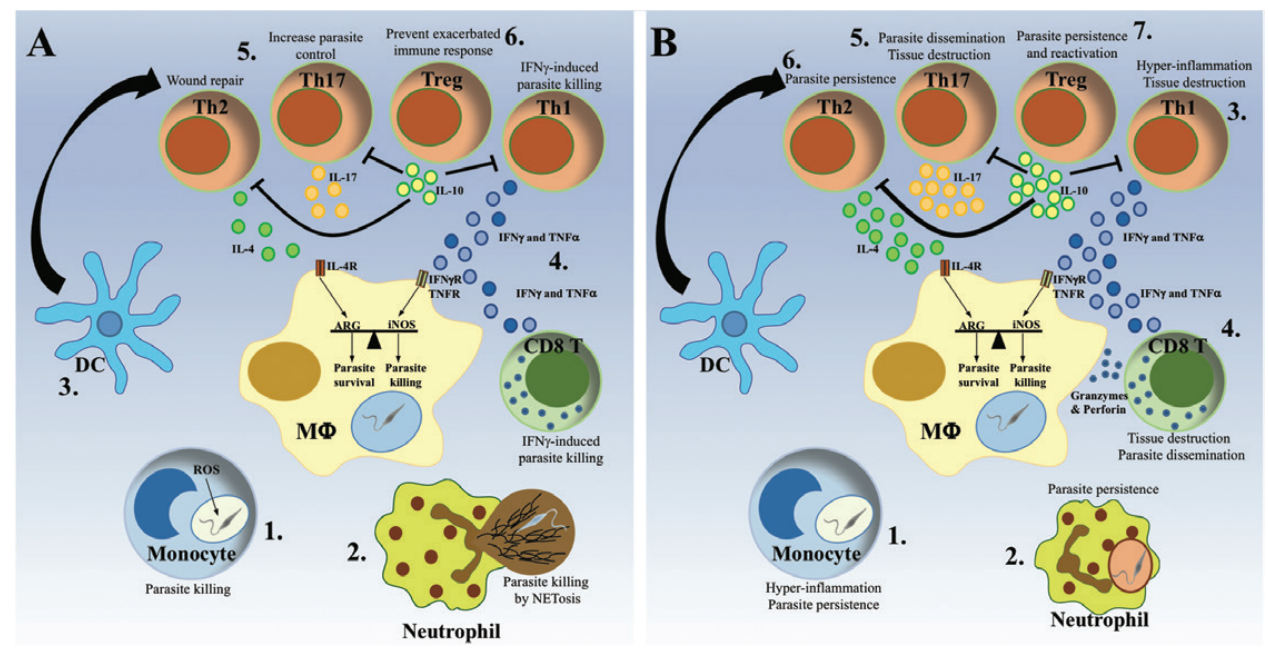

Fig. 3. Protective and pathological anti-Leishmania immune responses. The same cell types have been associated with either host protection or disease progression depending on parasite species and host immune-competence. (A) The protective immune response. Monocytes (1) and neutrophils (2) are rapidly recruited to the infection site, where they clear Leishmania parasites via ROS production or NET formation, respectively. Subsequently, dendritic cells (DC) migrate to the lymph node (3), where they activate and polarize T cells into different $T_{\text {n }}$ populations. The development of a predominant $\mathrm{T}_{h} 1$ immune response, characterized by production of IFN- $\gamma$ and TNF- $\alpha$ by CD4+ and CD8 $8^{+} \mathrm{T}^{+}$cells (4), activates infected macrophages to kill intracellular parasites via production of $\mathrm{NO}$, while a moderate $\mathrm{T}_{2} 2$ and $\mathrm{T}_{\mathrm{h}} 17$ response has been shown to promote the control of the parasite and the resolution of the infection (5). The subsequent development of IL-10-producing $T_{\text {reg }}$ cells ensures the dampening of the immune response, avoiding the development of chronic inflammation (6). (B) The pathological immune response. The excessive recruitment of cytokine-producing monocytes causes hyper-inflammation, which favors parasite persistence (1). Inefficient parasite killing by neutrophils hides parasites from macrophages, interfering with the development of the immune response and favoring parasite persistence (2). Exaggerated IFN- $\gamma$ and TNF- $\alpha$ production by CD4 ${ }^{+} \mathrm{T}_{\mathrm{h}} 1$ cells causes hyper-inflammation resulting in tissue destruction (3), whereas the addition of cytotoxic granzymes and perforin by $\mathrm{CD}^{+} \mathrm{T}$ cells promotes parasite dissemination (4). Similarly, a disproportionate $\mathrm{T}_{h} 17$ response induces tissue destruction and parasite dissemination, resulting in the formation of metastatic lesions (5). In contrast, an excessive T 2 response promotes parasite persistence and the development of chronic infection (6), while high numbers of IL-10-producing $T_{\text {reg }}$ cells promote parasite persistence and re-activation following the resolution of the disease (7).

exception of Leishmune® used for dogs. Also in this case, the central role of TLR stimulation in the determination of the immune response makes TLRs an important candidate not only for immunotherapy, but also for vaccine development. For instance, TLR4 and TLR9 ligands have been used as adjuvants in vaccine formulations, showing promising results in the murine model. In fact, the TLR4 agonist ONO-4007 was shown to boost the efficacy of $L$. amazonensis antigens, inducing protection in BALB/c mice, by promoting the production of IL-12 and IFN- $\gamma$ (97).

Furthermore, the TLR9 agonist CpG was used in many vaccine formulations and was shown to improve vaccine efficacy by promoting $\mathrm{IL}-12$ production, whereas the combination between TLR4 and TLR9 agonists increased their adjuvant potency, thus showing a synergizing effect between the two TLRs (98). Interestingly, mice immunized with a recombinant LRV capsid in combination with CpG are protected against the increased pathology of LRV-bearing L. guyanensis infection (99). Despite not conferring complete protection, LRV-based vaccination could be the first pre-emptive agent against disseminated leishmaniasis.

\section{Conclusions}

Here, we summarized some of the recent knowledge on the host immune response to Leishmania and the parasite immune evasion strategies. Several aspects of the antileishmanial immune response have been disclosed in the last 30 years. We now understand many more facets of the immune response to Leishmania than the oversimplified, bidimensional $\mathrm{T}_{h} 1 / \mathrm{T}_{h} 2$ dogma. Despite the increasing complexity of anti-leishmanial immunity, a mountain of data underline the importance of the balance between the different types of immune responses rather than the development of a unique and specific response (see Fig. 3).

Moreover, the involvement of bacterial and viral coinfection (often overlooked in the current murine models of infection) in the determination of the outcome of leishmaniasis highlights the need for new research models, better replicating the human immune system and taking into account the human microbiome which, on the basis of mouse models, is likely to be relevant to control or exacerbate Leishmania infection in the case of dysbiosis. Another aspect not to underestimate is the cohabitation between leishmaniasis and autoimmune disorders. The two diseases require completely opposite treatment strategies; thus the adjustment of an appropriate therapy could be particularly difficult, resulting in a vicious cycle of relapse of one or the other disease.

Despite the increased knowledge of the immunology of Leishmania infection, the treatment options for leishmaniasis are still limited and rely on old drugs often adopted and repurposed from other diseases, while an effective human vaccine has still to be developed. As the outcome of Leishmania infection is influenced by multiple factors, which could vary extremely between individuals, the use of 
immunotherapy to improve leishmanial treatment should also be adapted as a sort of precision medicine. Similarly, the parasite species specificity of the immune response required to clear Leishmania infection does not facilitate the generation of a universal vaccine. Thus, despite the recent discovery of an immunodominant antigen conserved in many Leishmania species (100), the combination with different adjuvants to develop many species-specific formulations could be required.

Finally, as the immune responses developed during the different types of leishmaniasis are drastically divergent, the generation of different outcome-specific anti-leishmanial treatments or vaccines might prove more effective than a unique solution for leishmaniasis.

\section{Funding}

This work was supported by the Swiss National Fund for Research (FNRS 310030-153204 and IZRJZ3_164176) and the Institute for Arthritis Research (iAR).

\section{Acknowledgements}

We are grateful to Dr Slavica Masina for critical reading of the manuscript. We would like to apologize to some of our colleagues for not being able to cite their work due to space constraints.

Conflicts of interest statement: The authors declared no conflicts of interest.

\section{References}

1 Alvar, J., Vélez, I. D., Bern, C. et al. WHO Leishmaniasis Control Team. 2012. Leishmaniasis worldwide and global estimates of its incidence. PLOS ONE 7:e35671.

2 Kaye, P. and Scott, P. 2011. Leishmaniasis: complexity at the hostpathogen interface. Nat. Rev. Microbiol. 9:604.

3 Scott, P. and Novais, F. O. 2016. Cutaneous leishmaniasis: immune responses in protection and pathogenesis. Nat. Rev. Immunol. 16:581.

4 Bourreau, E., Ginouves, M., Prévot, G. et al. 2016. Presence of Leishmania RNA virus 1 in Leishmania guyanensis increases the risk of first-line treatment failure and symptomatic relapse. J. Infect. Dis. 213:105.

5 Adaui, V., Lye, L. F., Akopyants, N. S. et al. 2016. Association of the endobiont double-stranded RNA virus LRV1 with treatment failure for human leishmaniasis caused by Leishmania braziliensis in Peru and Bolivia. J. Infect. Dis. 213:112.

6 Chagas, A. C., Oliveira, F., Debrabant, A., Valenzuela, J. G., Ribeiro, J. M. and Calvo, E. 2014. Lundep, a sand fly salivary endonuclease increases Leishmania parasite survival in neutrophils and inhibits XIla contact activation in human plasma. PLoS Pathog. 10:e1003923.

7 Franco, L. H., Beverley, S. M. and Zamboni, D. S. 2012. Innate immune activation and subversion of mammalian functions by Leishmania lipophosphoglycan. J. Parasitol. Res. 2012:165126.

8 Vinet, A. F., Fukuda, M., Turco, S. J. and Descoteaux, A. 2009. The Leishmania donovani lipophosphoglycan excludes the vesicular proton-ATPase from phagosomes by impairing the recruitment of synaptotagmin V. PLoS Pathog. 5:e1000628.

9 Iles, K. E. and Forman, H. J. 2002. Macrophage signaling and respiratory burst. Immunol. Res. 26:95.

10 Fang, F. C. 2004. Antimicrobial reactive oxygen and nitrogen species: concepts and controversies. Nat. Rev. Microbiol. 2:820.

11 Panday, A., Sahoo, M. K., Osorio, D. and Batra, S. 2015. NADPH oxidases: an overview from structure to innate immunityassociated pathologies. Cell. Mol. Immunol. 12:5.
12 Aktan, F. 2004. iNOS-mediated nitric oxide production and its regulation. Life Sci. 75:639.

13 Kima, P. E. 2007. The amastigote forms of Leishmania are experts at exploiting host cell processes to establish infection and persist. Int. J. Parasitol. 37:1087.

14 Carneiro, M. B. H., Roma, E. H., Ranson, A. J. et al. 2018. NOX2-derived reactive oxygen species control inflammation during Leishmania amazonensis infection by mediating infectioninduced neutrophil apoptosis. J. Immunol. 200:196.

15 Pham, N. K., Mouriz, J. and Kima, P. E. 2005. Leishmania pifanoi amastigotes avoid macrophage production of superoxide by inducing heme degradation. Infect. Immun. 73:8322.

16 Gaur, U., Roberts, S. C., Dalvi, R. P., Corraliza, I., Ullman, B. and Wilson, M. E. 2007. An effect of parasite-encoded arginase on the outcome of murine cutaneous leishmaniasis. J. Immunol. 179:8446.

17 Olivier, M., Atayde, V. D., Isnard, A., Hassani, K. and Shio, M. T. 2012. Leishmania virulence factors: focus on the metalloprotease GP63. Microbes Infect. 14:1377.

18 Khouri, R., Bafica, A., Silva, M. d. a. P. et al. 2009. IFN-beta impairs superoxide-dependent parasite killing in human macrophages: evidence for a deleterious role of SOD1 in cutaneous leishmaniasis. J. Immunol. 182:2525.

19 Ghosh, S., Goswami, S. and Adhya, S. 2003. Role of superoxide dismutase in survival of Leishmania within the macrophage. Biochem. J. 369(Pt 3):447.

20 Bose, M., Saha, R., Sen Santara, S., Mukherjee, S., Roy, J. and Adak, S. 2012. Protection against peroxynitrite by pseudoperoxidase from Leishmania major. Free Radic. Biol. Med. 53:1819.

21 Sansom, F. M., Tang, L., Ralton, J. E., Saunders, E. C., Naderer, T. and McConville, M. J. 2013. Leishmania major methionine sulfoxide reductase $A$ is required for resistance to oxidative stress and efficient replication in macrophages. PLOS ONE 8:e56064.

22 Hurrell, B. P., Regli, I. B. and Tacchini-Cottier, F. 2016. Different Leishmania species drive distinct neutrophil functions. Trends Parasitol. 32:392.

23 Gabriel, C., McMaster, W. R., Girard, D. and Descoteaux, A. 2010. Leishmania donovani promastigotes evade the antimicrobia activity of neutrophil extracellular traps. J. Immunol. 185:4319.

24 Hurrell, B. P., Schuster, S., Grün, E. et al. 2015. Rapid sequestration of Leishmania mexicana by neutrophils contributes to the development of chronic lesion. PLoS Pathog. 11:e1004929.

25 Crosby, E. J., Goldschmidt, M. H., Wherry, E. J. and Scott, P. 2014. Engagement of NKG2D on bystander memory CD8 T cells promotes increased immunopathology following Leishmania major infection. PLoS Pathog. 10:e1003970.

26 Goncalves, R., Zhang, X., Cohen, H., Debrabant, A. and Mosser D. M. 2011. Platelet activation attracts a subpopulation of effector monocytes to sites of Leishmania major infection. J. Exp. Med. 208:1253.

27 Romano, A., Carneiro, M. B. H., Doria, N. A. et al. 2017. Divergent roles for $\mathrm{Ly} 6 \mathrm{C}^{+} \mathrm{CCR} 2{ }^{+} \mathrm{CX} 3 \mathrm{CR} 1^{+}$inflammatory monocytes during primary or secondary infection of the skin with the intra-phagosomal pathogen Leishmania major. PLoS Pathog. 13:e1006479.

28 Glennie, N. D., Volk, S. W. and Scott, P. 2017. Skin-resident CD4+ $T$ cells protect against Leishmania major by recruiting and activating inflammatory monocytes. PLoS Pathog. 13:e1006349.

29 Xin, L., Vargas-Inchaustegui, D. A., Raimer, S. S. et al. 2010. Type I IFN receptor regulates neutrophil functions and innate immunity to Leishmania parasites. J. Immunol. 184:7047.

30 Figueira, C. P., Carvalhal, D. G., Almeida, R. A. et al. 2015 Leishmania infection modulates beta-1 integrin activation and alters the kinetics of monocyte spreading over fibronectin. Sci. Rep. 5:12862

31 Faria, M. S., Reis, F. C. and Lima, A. P. 2012. Toll-like receptors in Leishmania infections: guardians or promoters? J. Parasitol. Res. 2012:930257.

32 Tuon, F. F., Amato, V. S., Bacha, H. A., Almusawi, T., Duarte, M. I. and Amato Neto, V. 2008. Toll-like receptors and leishmaniasis. Infect. Immun. 76:866.

33 Becker, I., Salaiza, N., Aguirre, M. et al. 2003. Leishmania lipophosphoglycan (LPG) activates NK cells through Toll-like receptor-2. Mol. Biochem. Parasitol. 130:65. 
34 de Veer, M. J., Curtis, J. M., Baldwin, T. M. et al. 2003. MyD88 is essential for clearance of Leishmania major: possible role for lipophosphoglycan and Toll-like receptor 2 signaling. Eur. J. Immunol. 33:2822.

35 Bhattacharya, P., Bhattacharjee, S., Gupta, G. et al. 2010. Arabinosylated lipoarabinomannan-mediated protection in visceral leishmaniasis through up-regulation of Toll-like receptor 2 signaling: an immunoprophylactic approach. J. Infect. Dis. 202:145.

36 Kavoosi, G., Ardestani, S. K. and Kariminia, A. 2009. The involvement of TLR2 in cytokine and reactive oxygen species (ROS) production by PBMCs in response to Leishmania major phosphoglycans (PGs). Parasitology 136:1193.

37 Flandin, J. F., Chano, F. and Descoteaux, A. 2006. RNA interference reveals a role for TLR2 and TLR3 in the recognition of Leishmania donovani promastigotes by interferon-gamma-primed macrophages. Eur. J. Immunol. 36:411.

38 Ives, A., Ronet, C., Prevel, F. et al. 2011. Leishmania RNA virus controls the severity of mucocutaneous leishmaniasis. Science 331:775.

39 Ribeiro-Gomes, F. L., Moniz-de-Souza, M. C., Alexandre-Moreira, M. S. et al. 2007. Neutrophils activate macrophages for intracellular killing of Leishmania major through recruitment of TLR4 by neutrophil elastase. J. Immunol. 179:3988.

40 Faria, M. S., Reis, F. C., Azevedo-Pereira, R. L., Morrison, L. S., Mottram, J. C. and Lima, A. P. 2011. Leishmania inhibitor of serine peptidase 2 prevents TLR4 activation by neutrophil elastase promoting parasite survival in murine macrophages. J. Immunol. 186:411.

41 Filardy, A. A., Pires, D. R., Nunes, M. P. et al. 2010. Proinflammatory clearance of apoptotic neutrophils induces an IL-12(low) IL-10(high) regulatory phenotype in macrophages. J. Immunol. 185:2044.

42 Li, Y., Ishii, K., Hisaeda, H. et al. 2004. IL-18 gene therapy develops $T_{1}$ 1-type immune responses in Leishmania major-infected $\mathrm{BALB} / \mathrm{c}$ mice: is the effect mediated by the CpG signaling TLR9? Gene Ther. 11:941.

43 Liese, J., Schleicher, U. and Bogdan, C. 2007. TLR9 signaling is essential for the innate NK cell response in murine cutaneous Ieishmaniasis. Eur. J. Immunol. 37:3424.

44 Schleicher, U., Liese, J., Knippertz, I. et al. 2007. NK cell activation in visceral leishmaniasis requires TLR9, myeloid DCs, and IL-12, but is independent of plasmacytoid DCs. J. Exp. Med. 204:893.

45 Abou Fakher, F. H., Rachinel, N., Klimczak, M., Louis, J. and Doyen, N. 2009. TLR9-dependent activation of dendritic cells by DNA from Leishmania major favors $T_{h} 1$ cell development and the resolution of lesions. J. Immunol. 182:1386.

46 Ives, A., Masina, S., Castiglioni, P. et al. 2014. MyD88 and TLR9 dependent immune responses mediate resistance to Leishmania guyanensis infections, irrespective of Leishmania RNA virus burden. PLOS ONE 9:e96766.

47 Lima-Junior, D. S., Costa, D. L., Carregaro, V. et al. 2013. Inflammasome-derived IL-1 $\beta$ production induces nitric oxidemediated resistance to Leishmania. Nat. Med. 19:909.

48 Gupta, A. K., Ghosh, K., Palit, S., Barua, J., Das, P. K. and Ukil, A. 2017. Leishmania donovani inhibits inflammasome-dependent macrophage activation by exploiting the negative regulatory proteins A20 and UCP2. FASEB J. 31:5087.

49 Duong, B. H., Onizawa, M., Oses-Prieto, J. A. et al. 2015. A20 restricts ubiquitination of pro-interleukin-1 $\beta$ protein complexes and suppresses NLRP3 inflammasome activity. Immunity 42:55.

50 Charmoy, M., Hurrell, B. P., Romano, A. et al. 2016. The Nlrp3 inflammasome, IL-1 $\beta$, and neutrophil recruitment are required for susceptibility to a nonhealing strain of Leishmania major in C57BL/6 mice. Eur. J. Immunol. 46:897.

51 Lefèvre, L., Lugo-Villarino, G., Meunier, E. et al. 2013. The C-type lectin receptors dectin-1, MR, and SIGNR3 contribute both positively and negatively to the macrophage response to Leishmania infantum. Immunity 38:1038.

52 Iborra, S., Martínez-López, M., Cueto, F. J. et al. 2016. Leishmania uses mincle to target an inhibitory ITAM signaling pathway in dendritic cells that dampens adaptive immunity to infection. Immunity 45:788.

53 Silveira, F. T., Lainson, R., De Castro Gomes, C. M., Laurenti, M. D. and Corbett, C. E. 2009. Immunopathogenic competences of Leishmania (V.) braziliensis and L. (L.) amazonensis in American cutaneous leishmaniasis. Parasite Immunol. 31:423.

54 Vargas-Inchaustegui, D. A., Xin, L. and Soong, L. 2008. Leishmania braziliensis infection induces dendritic cell activation, ISG15 transcription, and the generation of protective immune responses. J. Immunol. 180:7537.

55 Agallou, M., Margaroni, M. and Karagouni, E. 2011. Cellular vaccination with bone marrow-derived dendritic cells pulsed with a peptide of Leishmania infantum KMP-11 and CpG oligonucleotides induces protection in a murine model of visceral leishmaniasis. Vaccine 29:5053.

56 Lopez Kostka, S., Dinges, S., Griewank, K., Iwakura, Y., Udey, M. C. and von Stebut, E. 2009. IL-17 promotes progression of cutaneous leishmaniasis in susceptible mice. J. Immunol. 182:3039.

57 Hartley, M. A., Bourreau, E., Rossi, M. et al. 2016. Leishmaniavirusdependent metastatic leishmaniasis is prevented by blocking IL-17A. PLoS Pathog. 12:e1005852.

58 Belkaid, Y., Piccirillo, C. A., Mendez, S., Shevach, E. M. and Sacks, D. L. 2002. $\mathrm{CD}^{+}{ }^{+} \mathrm{CD} 25^{+}$regulatory $\mathrm{T}$ cells control Leishmania major persistence and immunity. Nature 420:502.

59 Lages, C. S., Suffia, I., Velilla, P. A. et al. 2008. Functional regulatory $T$ cells accumulate in aged hosts and promote chronic infectious disease reactivation. J. Immunol. 181:1835

60 Bourreau, E., Prévot, G., Gardon, J., Pradinaud, R. and Launois, P. 2001. High intralesional interleukin-10 messenger RNA expression in localized cutaneous leishmaniasis is associated with unresponsiveness to treatment. J. Infect. Dis. 184:1628.

61 Katara, G. K., Ansari, N. A., Verma, S., Ramesh, V. and Salotra, P. 2011. Foxp3 and IL-10 expression correlates with parasite burden in lesional tissues of post kala azar dermal leishmaniasis (PKDL) patients. PLoS Negl. Trop. Dis. 5:e1171.

62 Stäger, S. and Rafati, S. 2012. CD8(+) T cells in leishmania infections: friends or foes? Front. Immunol. 3:5.

63 Uzonna, J. E., Joyce, K. L. and Scott, P. 2004. Low dose Leishmania major promotes a transient $\mathrm{T}$ helper cell type 2 response that is down-regulated by interferon gamma-producing CD8 ${ }^{+} \mathrm{T}$ cells. J. Exp. Med. 199:1559.

64 Nateghi Rostami, M., Keshavarz, H., Edalat, R., Sarrafnejad, A., Shahrestani, T., Mahboudi, F., and Khamesipour, A. 2010. CD8 ${ }^{+}$T cells as a source of IFN-gamma production in human cutaneous leishmaniasis. PLoS Negl. Trop. Dis. 4:e845.

65 Da-Cruz, A. M., Bertho, A. L., Oliveira-Neto, M. P. and Coutinho, S. G. 2005. Flow cytometric analysis of cellular infiltrate from American tegumentary leishmaniasis lesions. Br. J. Dermatol. 153:537.

66 Da-Cruz, A. M., Bittar, R., Mattos, M. et al. 2002. T-cell-mediated immune responses in patients with cutaneous or mucosal leishmaniasis: long-term evaluation after therapy. Clin. Diagn. Lab. Immunol. 9:251.

67 Bourreau, E., Ronet, C., Couppié, P., Sainte-Marie, D., TacchiniCottier, F. and Launois, P. 2007. IL-10 producing CD8 ${ }^{+}$T cells in human infection with Leishmania guyanensis. Microbes Infect. 9:1034.

68 Ganguly, S., Das, N. K., Panja, M. et al. 2008. Increased levels of interleukin-10 and IgG3 are hallmarks of Indian post-kala-azar dermal leishmaniasis. J. Infect. Dis. 197:1762.

69 Hernández-Ruiz, J., Salaiza-Suazo, N., Carrada, G. et al. 2010. CD8 cells of patients with diffuse cutaneous leishmaniasis display functional exhaustion: the latter is reversed, in vitro, by TLR2 agonists. PLoS Negl. Trop. Dis. 4:e871.

70 Faria, D. R., Souza, P. E., Duraes, F. V., Carvalho, E. M., Gollob, K. J., Machado, P. R. and Dutra, W. O. 2009. Recruitment of CD8(+) T cells expressing granzyme $A$ is associated with lesion progression in human cutaneous leishmaniasis. Parasite Immunol. 31:432.

71 Gaze, S. T., Dutra, W. O., Lessa, M. et al. 2006. Mucosal leishmaniasis patients display an activated inflammatory T-cell phenotype associated with a nonbalanced monocyte population. Scand. J. Immunol. 63:70. 
72 Novais, F. O., Carvalho, L. P., Graff, J. W. et al. 2013. Cytotoxic T cells mediate pathology and metastasis in cutaneous leishmaniasis. PLoS Pathog. 9:e1003504.

73 Crosby, E. J., Clark, M., Novais, F. O., Wherry, E. J. and Scott, P. 2015. Lymphocytic choriomeningitis virus expands a population of $\mathrm{NKG}_{2} \mathrm{D}^{+} \mathrm{CD}^{+} \mathrm{T}$ cells that exacerbates disease in mice coinfected with Leishmania major. J. Immunol. 195:3301.

74 Smelt, S. C., Cotterell, S. E., Engwerda, C. R. and Kaye, P. M. 2000. B cell-deficient mice are highly resistant to Leishmania donovani infection, but develop neutrophil-mediated tissue pathology. J. Immunol. 164:3681.

75 Wanasen, N., Xin, L. and Soong, L. 2008. Pathogenic role of B cells and antibodies in murine Leishmania amazonensis infection. Int. J. Parasitol. 38:417.

76 Silva-Barrios, S., Smans, M., Duerr, C. U. et al. 2016. Innate immune B cell activation by Leishmania donovani exacerbates disease and mediates hypergammaglobulinemia. Cell Rep. 15:2427

77 Eren, R. O., Reverte, M., Rossi, M. et al. 2016. Mammalian innate immune response to a Leishmania-resident RNA virus increases macrophage survival to promote parasite persistence. Cell Host Microbe 20:318.

78 Naik, S., Bouladoux, N., Wilhelm, C. etal. 2012. Compartmentalized control of skin immunity by resident commensals. Science 337:1115

79 Gimblet, C., Meisel, J. S., Loesche, M. A. et al. 2017. Cutaneous leishmaniasis induces a transmissible dysbiotic skin microbiota that promotes skin inflammation. Cell Host Microbe 22:13.e4.

80 Zangger, H., Hailu, A., Desponds, C. et al. 2014. Leishmania aethiopica field isolates bearing an endosymbiontic dsRNA virus induce pro-inflammatory cytokine response. PLoS Negl. Trop. Dis. 8:e2836

81 Rossi, M., Castiglioni, P., Hartley, M. A. et al. 2017. Type I interferons induced by endogenous or exogenous viral infections promote metastasis and relapse of leishmaniasis. Proc. Natl Acad. Sci. USA 114:4987.

82 Faucher, B., Bichaud, L., Charrel, R. et al. 2014. Presence of sandflies infected with Leishmania infantum and Massilia virus in the Marseille urban area. Clin. Microbiol. Infect. 20:0340.

83 Es-Sette, N., Ajaoud, M., Bichaud, L. et al. 2014. Phlebotomus sergenti a common vector of Leishmania tropica and Toscana virus in Morocco. J. Vector Borne Dis. 51:86.

84 Ergunay, K., Kasap, O. E., Orsten, S. et al. 2014. Phlebovirus and Leishmania detection in sandflies from eastern Thrace and northern Cyprus. Parasit. Vectors 7:575.

85 Sukla, S., Roy, S., Sundar, S. and Biswas, S. 2017. Leptomonas seymouri narna-like virus 1 and not leishmaniaviruses detected in kala-azar samples from India. Arch. Virol. 162:3827.

86 The PLOS Neglected Tropical Diseases Staff. 2015. Correction: Leishmania-HIV co-infection: clinical presentation and outcomes in an urban area in Brazil. PLoS Negl. Trop. Dis. 9:e0003653.
87 Parmentier, L., Cusini, A., Müller, N. et al. 2016. Severe cutaneous leishmaniasis in a human immunodeficiency virus patient coinfected with Leishmania braziliensis and its endosymbiotic virus. Am. J. Trop. Med. Hyg. 94:840

88 Zijlstra, E. E. 2014. PKDL and other dermal lesions in HIV coinfected patients with leishmaniasis: review of clinical presentation in relation to immune responses. PLOS Negl. Trop. Dis. 8:e3258.

89 Zijlstra, E. E., Musa, A. M., Khalil, E. A., el-Hassan, I. M. and el-Hassan, A. M. 2003. Post-kala-azar dermal leishmaniasis. Lancet Infect. Dis. 3:87

90 Wolday, D., Berhe, N., Britton, S. and Akuffo, H. 2000. HIV-1 alters $\mathrm{T}$ helper cytokines, interleukin-12 and interleukin-18 responses to the protozoan parasite Leishmania donovani. AIDS 14:921.

91 Kedzierska, K. and Crowe, S. M. 2002. The role of monocytes and macrophages in the pathogenesis of HIV-1 infection. Curr. Med. Chem 9:1893.

92 Shakya, N., Sane, S. A., Vishwakarma, P. and Gupta, S. 2012. Enhancement in therapeutic efficacy of miltefosine in combination with synthetic bacterial lipopeptide, Pam3Cys against experimental visceral leishmaniasis. Exp. Parasitol. 131:377.

93 Verthelyi, D., Gursel, M., Kenney, R. T. et al. 2003. CpG oligodeoxynucleotides protect normal and SIV-infected macaques from Leishmania infection. J. Immunol. 170:4717.

94 Miranda-Verástegui, C., Llanos-Cuentas, A., Arévalo, I., Ward B. J. and Matlashewski, G. 2005. Randomized, double-blind clinical trial of topical imiquimod $5 \%$ with parenteral meglumine antimoniate in the treatment of cutaneous leishmaniasis in Peru. Clin. Infect. Dis. 40:1395.

95 Machado, P. R., Lessa, H., Lessa, M. et al. 2007. Oral pentoxifylline combined with pentavalent antimony: a randomized trial for mucosal leishmaniasis. Clin. Infect. Dis. 44:788

96 Jeziorski, E., Dereure, J., Mac Bullen, G. et al. 2015. Mucosal relapse of visceral leishmaniasis in a child treated with antiTNF $\alpha$. Int. J. Infect. Dis. 33:135.

97 Calvopina, M., Barroso, P. A., Marco, J. D. et al. 2006. Efficacy of vaccination with a combination of Leishmania amastigote antigens and the lipid A-analogue ONO-4007 for immunoprophylaxis and immunotherapy against Leishmania amazonensis infection in a murine model of New World cutaneous leishmaniasis. Vaccine 24:5645

98 Raman, V. S., Bhatia, A., Picone, A. et al. 2010. Applying TLR synergy in immunotherapy: implications in cutaneous leishmaniasis. J. Immunol. 185:1701.

99 Castiglioni, P., Hartley, M. A., Rossi, M. et al. 2017. Exacerbated leishmaniasis caused by a viral endosymbiont can be prevented by immunization with its viral capsid. PLoS Negl. Trop. Dis. 11:e0005240.

100 Mou, Z., Li, J., Boussoffara, T. et al. 2015. Identification of broadly conserved cross-species protective Leishmania antigen and its responding CD4+ T cells. Sci. Transl. Med. 7:310ra167. 